\title{
Testing the Application of the Personalized Recommendation Method for Artworks
}

\author{
Miyoung Han \\ Hyung Jun Ahn*
}

School of Business, Hongik University, 94 Wausan-ro, Mapo-Gu, Seoul, Korea

*Corresponding author: hjahn@hongik.ac.kr

\section{Doi:10.5901/mjss.2015.v6n6s2p433}

\section{Abstract}

Rapid development of information technology is affecting not only business organizations but also museums of art as well. Many museums are trying to adopt new technologies so that the museums can enhance their interaction and shorten the distance with customers. Among various technologies, this research focuses on collaborative filtering which is one of the widely used methods of making personalized recommendation of products to customers online based on their preferences. While the technology has been successfully used in the e-commerce field for a decade, it is hard to find actual cases of its application in museums. Hence, this paper presents an effort to investigate whether collaborative filtering can be used effectively for fine art, and if so, what factors affect the effectiveness of its application. A survey was conducted to collect data about the participants' preferences of a range of fine art paintings. The data then were used to create preference estimations using the mechanism of collaborative filtering. The estimations were compared using different subsets of the data to identify which factors affect the accuracy of the preference estimation. It was found that collaborative filtering can successfully estimate the preference of the participants, and that the level of knowledge in art, prominence of artwork, and gender have significant effect on the accuracy of estimation.

Keywords: museum, museum 2.0, collaborative filtering, recommendation of artworks

\section{Introduction}

As predicted by Gordon Moore, the advancement of information technology continues to exhibit exponential growth, being arguably the most important driver of the changes in the society, economy, and culture across the globe (Brock \& Moore, 2006; Liddle, 2006). The dramatic difference between the everyday life of people today and that of a decade ago shows clearly how fast the changes have impacted every aspect of our life. Some of the most influential technologies today include Web 2.0, social media, and smart phones, just to name a few.

The changes have had the strongest impact on consumer products and e-commerce first. In contrast, the adoption of information technology has been rather slow in the field of arts and culture such as in museum management. Because of their roles of preserving traditional and cultural heritage, museums tend to exhibit conservative and inflexible organizational characteristics compared with the fields of commerce or popular culture, which often manifests through the rigid style of management. Moreover, it is known that the majority of the museums worldwide are usually unprofitable by themselves and need financial support from central or local governments, or otherwise, commercial organizations (Lindqvist, 2012; Rosenstein, 2010). This limitation often makes it more difficult for them to be active in adopting new technology.

Despite the difficulties, there is no doubt that museums can benefit from technology significantly in various ways (Besser, 1997). Information technology makes the collection, management, and sharing of information by museums much efficient, and can enable them overcome their physical limitation and reach more customers in a very interactive way. It can thus help museums shorten the distance with the customers and provide richer and novel services, possibly leading to improved profitability (Conwill \& Roosa, 2003; Hess et al., 2011).

From this background, this study focuses on collaborative filtering, which is one of the very successful technologies that have been utilized in the e-commerce field, to verify its applicability in museums of art. Collaborative filtering is used to make automated and personalized recommendation of products and services online using the past data about customers accumulated through various transactions. It has already been widely studied and successfully used for more than a decade in e-commerce (Ahn, 2006, 2008). The most well-known example of companies that utilize the technology is Amazon where the customers' behavior is constantly analyzed so that the products a user is most likely to prefer is 
recommended in various ways (Linden, Smith, \& York, 2003). Netflix is also well known for its system of recommending movies to customers based on their past viewing history or ratings of other movies (Bennett \& Lanning, 2007). Collaborative filtering works based on the similarity between customers or between products, where products preferred by similar customers, or products similar to already-purchased ones are recommended. The similarity is calculated with various measures such as the statistical correlation among people or products (Ahn, 2008).

By applying the technology, we can expect several benefits to museums and museumgoers. First, it can lower the search cost of customers significantly. Usually, many museums of art maintain a huge set of information about artwork, artists, exhibitions, or history of arts. Collaborative filtering can make the users' access to the information much easier by customizing the way information is found to each user. Second, it can be used as a way of enhancing interactivity between museums and people. Because collaborative filtering works by learning the preferences of the users from data, the relationship becomes mutual and evolving. That is, with more interaction between the users and the system of a museum, stronger relationship is built evolutionarily based on increasingly better understanding of the users. Third, it can be especially useful for people with insufficient knowledge in art. Based on statistical inference, collaborative filtering can possibly find the hidden preference of novices or beginners in arts and find artworks that are very likely to be preferred, which otherwise can be difficult without the help of experts. While experts' guidance is expensive or often unavailable, collaborative filtering can be used anytime at a very low cost once the system is built and running.

This study aims to test whether collaborative filtering can be successfully used for recommending artworks, and to investigate the factors that affect the effectiveness of it. For this research goal, first, a survey was conducted to collect the participants' preferences about artworks of different types. Then, the data were analyzed using collaborative filtering to test whether the mechanism can successfully estimate the participants' preferences of artworks. If the estimation is effective, the result can be directly used for personalized recommendation of artworks. It was also tested whether some factors have significant influence on the accuracy of estimation. The factors were drawn from the review of previous literature.

The rest of the paper is structured as follows. First, the next section briefly reviews the background of research. The third section presents research hypotheses. The fourth section shows data collection, research method, and analysis results. The fifth section concludes with summary, discussion, and further research issues.

\section{Research Background}

\subsection{Museum and information technology}

Like all the other areas, museums are under the inevitable influence of information technology. The traditional roles of a museum consist of collection, preservation, research, exhibition, and education (Anderson, 2004; Hooper-Greenhill, 1999). Technology not only is a tool that supports these traditional roles, but can also be used to bring more fundamental and radical changes to museums.

One of the most notable roles of museums in the eyes of museumgoers is their role as a showroom of artworks. That is, typical museums showcase selected pieces of art for visitors' appreciation. Compared with the traditional style of exhibition, technology can help museums provide much richer information to visitors, far beyond what traditional exhibitions can offer. This includes information about individual artworks, artists, reviews, exhibitions, and historical information. Although all these can be provided through traditional media as well such as paper-based pamphlets or offline education, information technology makes it much easier to both provide and access the information. The information can also be easily enriched with audio, video, or hyper-linked texts at a very low cost. This also implies that the information that has been limitedly accessible to experts or heavy hobbyists can now be made easily accessible to a much wider audience thanks to technology.

Another notable change is the advent of virtual museums. As the name suggests, virtual museums are online venues for exhibition of artworks with which both museums and users can overcome many of the limitations of the traditional space of museums (Rayward \& Twidale, 1999). Many museums maintain larger collections of items than those on physical display, and thus, only part of what a museum owns is usually accessible to people at any given time. For reasons of security and protection of the items on display, visitors are allowed only restricted ways of experiencing the artworks from a certain physical distance. That is, viewers cannot touch or get too close to the items, nor can they view the items from various angles. Virtual museums can overcome these limitations effectively thanks to various technologies such as virtual reality, augmented reality, and high-definition displays easily available and affordable these days. For example, Google Art Project provides access to various exhibitions of more than 150 partner museums in about 40 countries with very high-resolution images of artworks (Proctor, 2011). Some of the virtual exhibitions even enable walk- 
through of the virtual space using Google's street view technology. With this, users can literally walk around the virtual exhibition room, and zoom in and out to the artworks for a very up-close viewing which often even exceeds the level of detail perceivable in offline experience.

Information technology can also easily turn the traditional one-way experience of museums into a two-way or participatory one. Most museums have traditionally let visitors passively follow the paths of movement to view the items on display in a way rather strictly planned by their curators. Thus, viewers are only given the role as the consumer of the exhibition, with little room for interactive participation or two-way communication in the exhibition. Information technology provides various ways with which museums can become more interactive. For example, an exhibition can be designed based on a simple voting on a social network service, where the participants choose which artworks or artists are to be included in the exhibition (Simon, 2010). At Tate Modern museum, children are encouraged to use a touch-based device installed at the museum to produce a creative digital painting, which is collaged on a big screen as a part of its constant and evolving exhibition (Johanson \& Glow, 2012). Another example is the "This Exquisite Forest" project, which is an evolutionary and collaborative Web 2.0 platform of creating animations where participants from around the world can collectively contribute to a gradually growing online animation (Koblin \& Milk, 2013; Literat, 2012).

All in all, it is generally agreed that museums are also at a turning point for big changes. The changes can enable visitors to experience museums in far more various ways than before. However, it appears that the traditional conservative nature of museum organizations make it hard for them to adapt to the changes. With this background, this study aims to test collaborative filtering, one of the very successful technologies that has been used widely in the ecommerce field, to see whether it can be successfully applied to museums.

\subsection{Personalization for museums}

Personalization refers to the services that aim to provide tailored products, information, or advertisement to meet the unique preferences of individual users (Ahn, 2008; Komiak \& Benbasat, 2006). Although the concept has been around for a very long time in marketing literature, it is the Internet and e-commerce that has enabled many companies to implement it practically. The reason is mainly because customizing information contents, user interfaces, or business processes online is much easier using software compared with the traditional transaction environment in the physical world.

Many earlier personalization methods relied on rule-based systems, where a user's choice of options creates rules, and the rules are programmed as software for providing personalized services. However, recent personalization systems mainly rely on collaborative filtering (CF) (Ahn, 2008; Bobadilla, Ortega, \& Hernando, 2012). CF utilizes the ratings of users on various products or services. The ratings are analyzed to infer the preferences of a user for items that the user has not yet purchased or shown interests in. That is, the ratings of all the users available are utilized for filtering the products for a specific user. This process can be described as a virtual collaboration among the users, and hence, it is called collaborative filtering. This study also utilizes the mechanism of CF. The mechanism is briefly illustrated in Figure1.

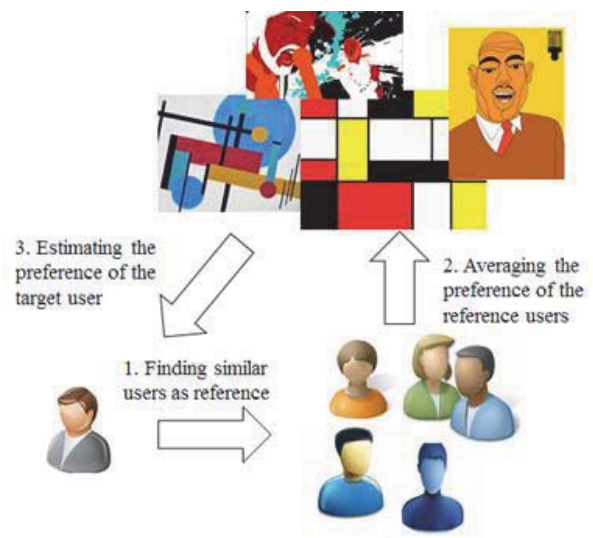

[Figure 1]. Brief Illustration of Collaborative Filtering

Application of personalization in the field of artwork is at an early stage, and there have been few example cases of it 
reported yet. One notable example is the Art Recommender system of Rijksmuseum in Amsterdam (Wang et al., 2008), which is known to utilize the content-based approach of personalization. Different from collaborative filtering, contentbased approaches use content information that include the title, description, keywords, or others to match items for recommendation (Burke, 2002). Based on the inputs from a user, the system recommends paintings of the museum, and also, several possible routes of tour inside the museum that allows the user to view all the recommended paintings. Users can upload the routes on his or her mobile device, and follow one of them in their actual visits to make sure they do not miss any of the paintings recommended by the system.

Considering the strengths of collaborative filtering as described above, this study aims to test the application of it to the museums of fine art. While doing so, this study also tries to test the factors that may impact its accuracy when applied to artworks.

\section{Research Objective and Hypotheses}

\subsection{Research Objectives}

This study has several objectives. First, as introduced in the previous section, this study tries to test the usability of personalization based on collaborative filtering for artworks by applying it to the field. Second, this study aims to test several factors that can possibly influence the effectiveness of collaborative filtering when applied to artworks. Especially, this study will focus on some personal characteristics that have already been studied in the e-commerce field. Considering that the personal factors such as experience and knowledge influence the museum experience of individual visitors, investigating the effect of such factors on personalization is also very important (Tallon, 2008).

\subsection{Can collaborative filtering work for artworks?}

The consumption of fine art is often distinguished from that of ordinary consumer products. For most people, fine art is experienced without purchase. Different from usual consumer products, the value of an artwork is not determined by its practical utility, but more by its artistic inspiration and symbolic values (Findlay, 2014; Heilbrun \& Gray, 2001). Considering these differences, consumer experience of fine art can be regarded as being more similar to that of movies or popular music to a certain degree. Luckily, many studies have already been conducted about movies and pop-music in the e-commerce field, mainly thanks to the abundance and accessibility of online data about movies and music. Collaborative filtering has been effectively applied to the datasets of movies and music in numerous studies (Ahn, 2006; Hayes \& Cunningham, 2004), suggesting the possibility of its application in the field of fine art as well.

For collaborative filtering to work effectively for artworks, the mechanism needs to identify different groups of people with disparate preferences for artworks. For example, if we can find a consistent tendency of a group of people to like the artworks of impressionism, we can easily recommend artworks of the sorts to those individuals. Assuming this is possible considering the similarity between the fields such as discussed above, the following is postulated:

Hypothesis 1: Collaborative filtering can effectively estimate the preferences of people for fine art.

\subsection{Level of Knowledge}

The next hypotheses are about the individual characteristics that may affect the accuracy of collaborative filtering. According to many previous studies in the e-commerce field, the effectiveness of collaborative filtering can vary according to individual characteristics (Ahn \& Park, 2012; Bettman \& Park, 1980). Simply, collaborative filtering can be more effective for some people than the others.

The knowledge of a user is one of the many personal factors known to have a strong impact on the effectiveness of collaborative filtering (Bettman \& Park, 1980; Hong \& Sternthal, 2010). In the previous studies, it was found that the prior knowledge influences the overall preference of a product, search behavior, and the style of information processing (Ahn \& Park, 2012; Brucks, 1985; Su, Comer, \& Lee, 2008). Especially, studies have revealed that customers with higher level of knowledge in products can process more complex information and have a higher understanding of individual product attributes (Ahn \& Park, 2012; Su et al., 2008). A study about MP3 players also showed that the level of knowledge about the devices affects how easy it is for the person to use a recommendation system (Su et al., 2008). Another study by Brucks (1985) showed that prior knowledge makes search easier (Brucks, 1985), which makes knowledgeable customers less dependent upon customer-aid tools such as recommendation systems.

The above results can be extended to the field of fine art. It can be reasoned that it is more difficult to estimate the 
preferences of those users with a higher level of knowledge in fine art because collaborative filtering estimates the preference based on the similarity between users. That is, it can be difficult to find a group of similar users for an expert user because of the user's sophisticated and differentiated preferences about artworks. On the other hand, because the lower level of knowledge in art can make it difficult to comprehend the complicated attributes of artworks and develop very unique preferences about them, it can be relatively easier to find a group of similar users for those non-experts. Therefore, it is postulated that the mechanism of collaborative filtering can more accurately estimate the preference of the users with a lower-level of knowledge based on the similarity.

Hypothesis 2-1: The accuracy of preference estimation by collaborative filtering is higher for users with lower level of knowledge in art.

The same logic can also be applied to the prominence of individual artworks or artists. It can be argued that people can generally develop more differentiated and unique preferences for relatively prominent artworks since people have relatively good knowledge of them. The differentiated preferences can again make it difficult to find similarities and make recommendation. Previous studies in the e-commerce field have also shown the impact of product-level popularity on recommendation (Ahn, 2006; Xiao \& Benbasat, 2007). Therefore, the followings are proposed:

Hypothesis 2-2: The accuracy of preference estimation by collaborative filtering is higher for less-known artworks. Hypothesis 2-3: The accuracy of preference estimation by collaborative filtering is higher for less-known artists.

\subsection{Gender}

Gender has long been the subject of study in marketing. Many concepts such as gender identity, biological sex, and gender role attitude have been found to influence customer behavior and decision making (Fischer \& Arnold, 1994). In the e-commerce field, studies have found that gender difference can affect how dependent a customer is on others, and also how active a customer is in adopting and buying with new technology such as collaborative filtering (Bae \& Lee, 2010; McMahan, Hovland, \& McMillan, 2009).

Gender is also known to affect the effectiveness of recommendation systems. According to Chang and Chin (2010), male participants of the study appeared to use recommendation systems more frequently compared with female (Chang \& Chin, 2010). Other studies similarly showed that gender affects the effectiveness and acceptance of recommendation systems. For example, in the case of an experiment of recommending cameras in an online store, the attributes of a camera preferred by one gender appeared to be very different from the ones favored by the other, hence affecting the acceptance of a recommendation system depending on its selective support of the attributes (Xiao \& Benbasat, 2007).

The difference by gender is also apparent in the field of art and culture. It is generally accepted that women on average are more familiar with art. It is known that women have more interest in art, and visit art museums more often (Lizardo, 2006). It is also known that genetic differences such as the cognitive ability of handling visual stimulus also make women more capable of appreciating artwork (Abramov, Gordon, Feldman, \& Chavarga, 2012; Fischer \& Arnold, 1994). In short, it can be postulated that both the biological and experiential differences make women more likely to develop advanced and individual preferences for fine art. Thus, also drawing on the assumption of hypothesis 2-1, the following is proposed:

Hypothesis 3: The accuracy of preference estimation by collaborative filtering is higher for male compared with female.

\section{Data Collection and Analysis}

\subsection{Data Collection}

Data were collected through a survey of 112 university students who do not major in art or art-related fields. 69 of them were female and 43 male. The respondents rated 100 paintings of fine art in the scale of 1 to 5 representing how much they preferred each, where 5 means most preferred. The survey also measured the participants' general level of knowledge in art, of which the median value was used for dividing the participants into two groups of higher and lowerlevel knowledge in art. The survey instruments were adapted from a previous study (Czellar \& Luna, 2010), and are shown in Table 1.

The 100 paintings were selected so that 40 of them were well-known artworks and the others not. Selection of the well-known artworks and artists was based on whether they have appeared in the text books of the elementary schools in Korea, or whether they have been included in the exhibition in the past by the major museums of Korea that include 
National Museum, National Museum of Modern and Contemporary Art, and Seoul Museum of Art.

[Table 1]. Instruments used for the level of knowledge in art

\begin{tabular}{c|l}
\hline No & Survey Instrument \\
\hline 1 & I am familiar with fine art. \\
2 & I know fine art well. \\
3 & I have higher level of knowledge in fine art compared with average people. \\
4 & I know the important factors that affect my satisfaction of an art exhibition. \\
\hline
\end{tabular}

Table 2 shows the composition of the paintings used for the survey. The study included both Korean and Western paintings by both well-known and less-known artists. Note that the western paintings of well-known artists were further divided into well-known and less-known artworks according to the criterion explained earlier, which was for testing hypothesis 4 . Figure 2 shows some examples of the paintings.

[Table 2]. Number of Paintings Used for the Survey

\begin{tabular}{l|lcc}
\hline Korean/Western & Prominence & No. of paintings & Category ID \\
\hline Korean & Works of well-known artists & 15 & 1 \\
\multirow{4}{*}{ Western } & Works of less-known artists & 10 & 2 \\
& Well-known works of well-known artists & 25 & 3 \\
& Less-known works of well-known artists & 25 & 4 \\
& Works of less-known artists & 25 & 5 \\
& Total & 100 & \\
\hline
\end{tabular}
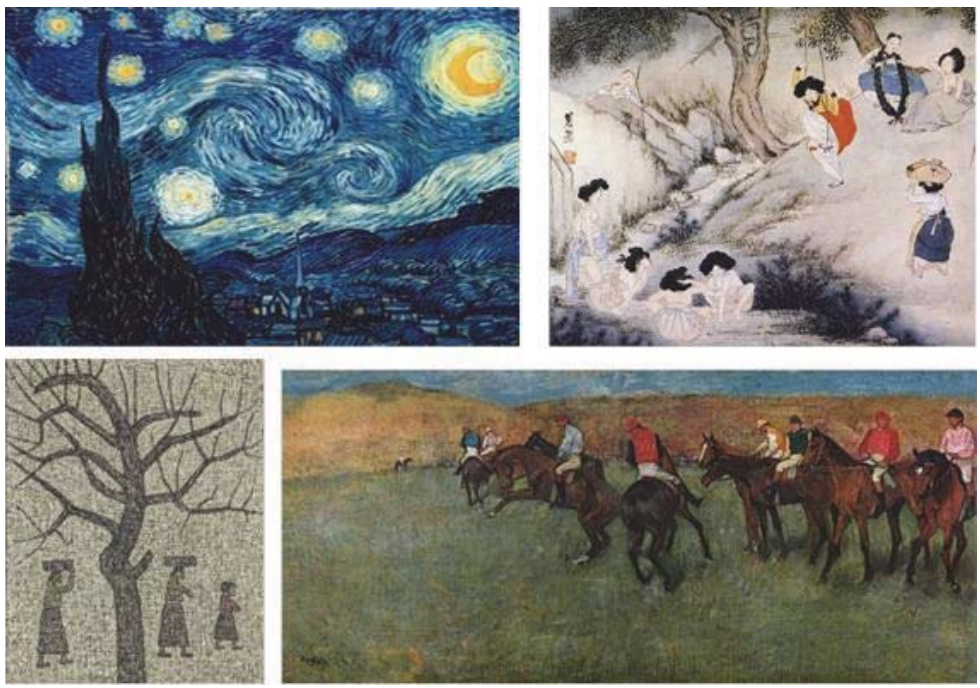

[Figure 2]. Sample Paintings Used for the Survey

\subsection{Analysis}

The data were analyzed as follows:

1. Paintings are split into two sets. One set is used for training, and the other for testing. That is, as shown in Figure 3 , half of the data is used to estimate the preference of the participants, and the other half is used for testing how accurate the estimation is.

2. The estimation is conducted for each group defined by each hypothesis. For example, for hypothesis 2-1, the estimation is conducted for the two groups of higher and lower-level knowledge in art. 
3. The estimation accuracy is compared among the groups to test each hypothesis. That is, for hypothesis $2-1$, it is statistically tested whether the accuracy is higher for the group of lower-level knowledge in art.

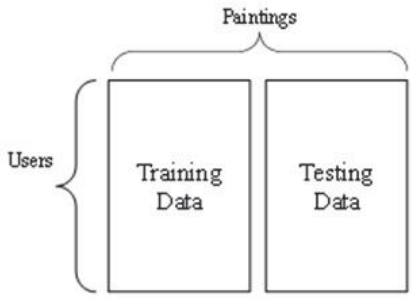

[Figure 3]. Usage of the Ratings Matrix (User $\times$ Painting)

For measuring the accuracy of estimation, mean absolute error (MAE) was used for its popularity in collaborating filtering research. The following formula was used for estimating the preference of the participants, which is called item-based collaborative filtering (IBCF) because it utilizes the similarity between items, instead of the similarity between users. IBCF was chosen because it has been used widely, and is known to outperform user-based collaborative filtering (UBCF) (Ahn, 2008). In short, the mechanism estimates the preference of a target user using the user's preference of other objects, in this case, artworks.

$$
p_{u, i}=\frac{\sum_{j \in \text { all_rated_items }} S_{i, j} \cdot R_{u, j}}{\sum_{j \in \text { all_rated_items }}\left|S_{i, j}\right|}
$$

In the formula, the preference of item $i$ by user $u, p_{u, i}$ is calculated as the weighted average of the ratings denoted as $R_{u, j}$ of the user for other already-rated items. The similarity $S_{i, j}$ is calculated using Pearson's correlation between item $i$ and $j$ as shown below, where $U$ represents the collection of all the users for whom the ratings for both $i$ and $j$ are known.

$$
S_{i, j}=\frac{\sqrt{\sum_{u \in U}\left(R_{u, i}-\bar{R}_{i}\right)\left(R_{u, j}-\bar{R}_{j}\right)}}{\sqrt{\sum_{u \in U}\left(R_{u, i}-\bar{R}_{i}\right)^{2}} \sqrt{\sum_{u \in U}\left(R_{u, j}-\bar{R}_{j}\right)^{2}}}
$$

\subsection{Testing the hypotheses}

For testing hypothesis 1, the collaborative filtering method was compared with three other strategies, namely random, user average, and artwork average strategies. Random strategy estimates the preferences randomly for each rating of each user, and hence, is the baseline strategy. User Average strategy uses the average rating of a given user as the estimation of all the ratings of the user. Artwork Average similarly uses the average rating of an artwork for the estimation of the ratings of the artwork by all users. As shown in Figure 4, the collaborative filtering strategy shows the smallest estimation error. A pairwise t-test was conducted to verify the statistical significance of the difference between CF and the user average strategy which exhibited the next best accuracy. Result of the one-tailed test revealed the $p$ value $<0.001$, confirming that the dominant result of CF is statistically significant. Hence, hypothesis 1 was accepted.

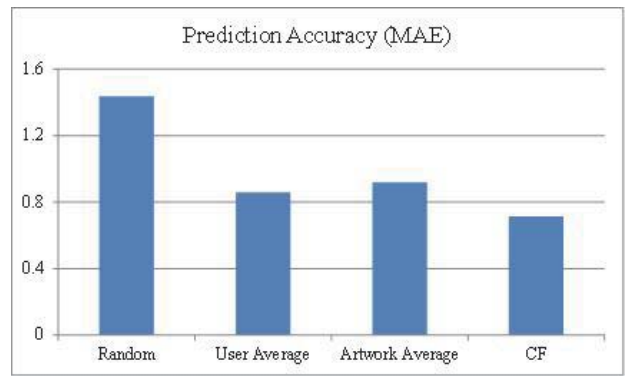

[Figure 4]. Hypothesis 1: Comparing the accuracy of CF against baseline strategies 
For hypotheses 2 to 3 , data were split into two groups for comparison according to each hypothesis. Table 3 shows the result of the t-test comparing the two groups of higher and lower-level of knowledge in art. It can be seen that it is more accurate for the lower level group with significance level $p<0.1$.

[Table 3]. Hypothesis 2-1: Level of knowledge in art

\begin{tabular}{lcccc}
\hline Group & Mean & Samples & $\mathrm{t}$ & $\mathrm{p}$ \\
\hline Higher-level of knowledge & 0.7386 & 58 & \multirow{2}{*}{1.45262} & \multirow{2}{*}{0.074575} \\
\hline Lower-level of knowledge & 0.6928 & 55 & & \\
\hline
\end{tabular}

The difference between well-known and less-known artworks also appeared to be significant (Table 4). The test was conducted between the paintings group 3 and 4 of the test data. Despite the limited number of samples in the comparison, the result showed significant difference $(p<0.05)$, confirming that the accuracy can be higher for less-known paintings.

[Table 4]. Hypothesis 2-2: Prominence of artwork

\begin{tabular}{lcccc}
\hline Group & Mean & Samples & $\mathrm{t}$ & $\mathrm{p}$ \\
\hline Well-known & 0.7682 & 12 & 1.746217 & 0.047059 \\
\hline Less-known & 0.6999 & 13 & & \\
\hline
\end{tabular}

Result of testing hypothesis 5 turned out to be insignificant (Table 5). The comparison was made between the paintings group 1,3 , and 4 versus 2 and 5 of the test data.

[Table 5]. Hypothesis 2-3: Prominence of artists

\begin{tabular}{lcccc}
\hline Group & Mean & Samples & $\mathrm{t}$ & $\mathrm{p}$ \\
\hline Well-known & 0.7208 & 32 & \multirow{2}{*}{1.677224} & 0.277757 \\
\hline Less-known & 0.7050 & 18 & & \\
\hline
\end{tabular}

Next, Table 6 shows the result for hypothesis 3 about gender. The result also confirms the hypothesis at the significance level $p<0.05$, showing that the estimation by CF can be more accurate for male.

[Table 6]. Hypothesis 3: Gender

\begin{tabular}{lcccc}
\hline Group & Mean & Samples & $\mathrm{t}$ & $\mathrm{p}$ \\
\hline Male & 0.6933 & 69 & -1.754905 & 0.041339 \\
\hline Female & 0.7501 & 43 & & \\
\hline
\end{tabular}

\section{Conclusion}

\subsection{Summary of the result}

This study tested the possibility of applying the CF method, which is one of the most popular methods of personalization in the e-commerce field, to the museums of fine art. For the study, a survey was conducted to collect the data about the participants' preferences of artworks with different characteristics. The data were used for testing the mechanism of CF in order to investigate whether CF can be successfully used for artworks, and to find the factors that influence the accuracy of CF for museums of art.

The findings can be summarized as follows. Compared with several baseline strategies of recommendation, CF appeared to be more accurate in estimating the preference of the participants' preferences. In other words, this confirms the first hypothesis that CF can be successfully used for artworks. Second, CF showed higher accuracy for the participants with lower-level of knowledge, and for male participants compared with female. The result was consistent with the hypotheses that assumed having less knowledge and experience makes it difficult to develop more diversified 
and sophisticated preference, which in turn makes it easy for CF to estimate their preferences. Next, the hypotheses about the prominence of the artworks and artists were also tested. The result was significant for the artwork, showing that less prominence of the artworks leads to higher accuracy of CF. However, it was insignificant for the artists. The result may be due to the unpopularity of some of the artworks by popular artists used in the experiment. That is, because not all the paintings of famous artists are known very widely to public, the participants of the survey may not have developed specific preferences of them. We can also infer that the variations of the attributes of the artworks of the same artist are large enough for public that it is not likely for them to exhibit very consistent preferences for them.

\section{Discussion and Conclusion}

The following summarizes the academic contribution of the research. First, this study is one of the earliest studies that show that the personalized recommendation can be successfully used in the field of fine art. To the authors' best knowledge, it is very hard to find studies on this subject except for a very few experimental web sites. None of them appear to apply the popular and effective mechanism of recommendation such as collaborative filtering used in this study. Second, this study showed that there are some factors that can contribute to the effectiveness of personalized recommendation. It was shown that recommendation accuracy could be higher for male, people with lower-level of knowledge in art, and for less-known artworks, which lays a foundation for further research on this issue for exploring more factors that can be used for personalized recommendation. Third, although there have been abundant studies that present just ideas or conceptual suggestions of using technology for museums, it is hard to find academic studies that actually experiment or apply information technology for the field. With the growing needs and importance of such practical application of technology, this research can be considered as filling the gap.

The study provides several practical implications for museums and art-related businesses as well. First, the proposed mechanism can readily be utilized by museums. Considering the growing importance of the role of museums as information provider, being able to supply personalized or custom-tailored information can help museums offer much more satisfactory experience to visitors. Since museum experience is affected by various personal and contextual variables, provision of indiscriminate information or advertisement to all visitors cannot influence them effectively, often being regarded as spamming. Using collaborative filtering, a museum can, for example, send tailored information via email or Twitter about only the exhibitions or the artists that a given user is highly likely to prefer. Second, this study showed that the effect of personalization can vary across people. This result is useful since museums can take different approaches for distinct target groups. On the one hand, a museum can first choose to focus its effort of applying CF on the group of visitors for whom personalization can be more effective. For instance, museums can first target novice visitors for more successful results of applying CF, which can help them achieve early success. On the other hand, museums can take a non-public strategy for marketing to expert user groups, such as providing more specialized and higher-level knowledge to the group, rather than trying to extract public and common interests from them through collaborative filtering. Third, CF can not only be used as a tool for recommendation, but as a platform for building evolving relationship with visitors based on the accumulated understanding of them. Museums can go through a loop of providing personalized information, collecting users' feedback for the personalization, and enhancing the personalization based on the feedback. Through this iterative process, museums can persistently improve their understanding of the visitors.

The study has a couple of limitations and further research issues. First, the study was conducted on a relatively small and homogeneous group of participants. This limits the generalization of the results, and also requires further study involving larger and more different groups of participants. Second, further studies can consider other types of recommendation methods to possibly find out more effective approach of personalized recommendation for artworks. Other approaches in literature can be applied and tested, and the accuracy of preference estimation should be compared.

\section{References}

Abramov, I., Gordon, J., Feldman, O., \& Chavarga, A. (2012). Sex and vision II: color appearance of monochromatic lights. Biology of Sex Differences, 3(1), 1-15.

Ahn, H. J. (2006). Utilizing Popularity Characteristics for Product Recommendation. International Journal of Electronic Commerce, 11(2), 57-78.

Ahn, H. J. (2008). A new similarity measure for collaborative filtering to alleviate the new user cold-starting problem. Information Sciences, 178(1), 37-51.

Ahn, H. J., \& Park, S. (2012). How does customer's product expertise moderate the usefulness of information recommendation agents in online stores? Information Research, 17(4). Retrieved from http://search.ebscohost.com/login.aspx?direct=true\&profile=ehost\& scope=site\&authtype=crawler\&jrnl=13681613\&AN=97202400\&h=FwkCu9CI98nZYGd\%2F6y\%2BI\%2FHVG93A9\%2BrscS9PJC 
MCbBSEUYpmS9zXh6lz67ZTbrdN1SbeQFEStPdubn5g7w8v5bg\%3D\%3D\&crl=c

Anderson, G. (2004). Reinventing the museum: Historical and contemporary perspectives on the paradigm shift. Rowman Altamira. Retrieved from https://books.google.at/books?hl=ko\&lr=\&id=cjw-x3ZIOXUC\&oi=fnd\&pg=PP2\&dq=role+of+museum\&ots=7BLkR E6rZ6\&sig=EvCJvKyeFf6gyqVXoBkekN2FTRI

Bae, S., \& Lee, T. (2010). Gender differences in consumers' perception of online consumer reviews. Electronic Commerce Research, 11(2), 201-214. http://doi.org/10.1007/s10660-010-9072-y

Bennett, J., \& Lanning, S. (2007). The netflix prize. In KDD Cup and Workshop (Vol. 2007).

Besser, H. (1997). The transformation of the museum and the way it's perceived. The Wired Museum: Emerging Technology and Changing Paradigms, 153-170.

Bettman, J. R., \& Park, C. W. (1980). Effects of Prior Knowledge and Experience and Phase of the Choice Process on Consumer Decision Processes: A Protocol Analysis. Journal of Consumer Research, 7(3), 234-248. http://doi.org/10.2307/2489009

Bobadilla, J., Ortega, F., \& Hernando, A. (2012). A collaborative filtering similarity measure based on singularities. Information Processing \& Management, 48(2), 204-217.

Brock, D. C., \& Moore, G. E. (2006). Understanding Moore's law: four decades of innovation. Chemical Heritage Foundation. Retrieved from https://books.google.at/books?hl=ko\&lr=\&id=woBkE-_SOCUC\&oi=fnd\&pg=PR9\&dq=\%22moore\%27s+law\%22+art+and+ culture\&ots=XFZvzsdH8n\&sig=kibnrWcTt64t54anHAIBE2PVQ̄Qk

Brucks, M. (1985). The Effects of Product Class Knowledge on Information Search Behavior. Journal of Consumer Research, 12(1), 116. http://doi.org/10.2307/2489377

Burke, R. (2002). Hybrid Recommender Systems: Survey and Experiments. User Modeling and User-Adapted Interaction, 12(4), 331370 .

Chang, C.-C., \& Chin, Y.-C. (2010). The impact of recommendation sources on online purchase intentions: The moderating effects of gender and perceived risk. World Academy of Science, Engineering and Technology, 66, 111-114.

Conwill, K., \& Roosa, A. (2003). Cultivating community connections (Museums encouraged to pursue their potential as active, visible players in community life). Museum News, 82(3), 40-47.

Czellar, S., \& Luna, D. (2010). The effect of expertise on the relation between implicit and explicit attitude measures: An information availability/accessibility perspective. Journal of Consumer Psychology, 20(3), 259-273.

Findlay, M. (2014). The Value of Art: Money, Power, Beauty (Revised edition). Munich: Prestel USA.

Fischer, E., \& Arnold, S. J. (1994). Sex, gender identity, gender role attitudes, and consumer behavior. Psychology and Marketing, 11(2), 163-182. http://doi.org/10.1002/mar.4220110206

Hayes, C., \& Cunningham, P. (2004). Context boosting collaborative recommendations. Knowledge-Based Systems, 17(2-4), 131-138.

Heilbrun, J., \& Gray, C. M. (2001). The Economics of Art and Culture (2 edition). New York: Cambridge University Press.

Hess, M., Millar, F. S., Robson, S., MacDonald, S., Were, G., \& Brown, I. (2011). Well Connected to Your Digital Object? E-Curator: A Web-based e-Science Platform for Museum Artefacts. Literary and Linguistic Computing, 26(2), 193-215.

Hong, J., \& Sternthal, B. (2010). The Effects of Consumer Prior Knowledge and Processing Strategies on Judgments. Journal of Marketing Research, 47(2), 301-311. http://doi.org/10.1509/jmkr.47.2.301

Hooper-Greenhill, E. (1999). The educational role of the museum. Psychology Press. Retrieved from https://books.google.at/books?hl= ko\&lr=\&id=-3_9K-TcPiwC\&oi=fnd\&pg=PR10\&dq=role+of+museum\&ots=0RBzc2NCLs\&sig=L-zeXouxguwoVEVJoDr6qiT4Sdw

Johanson, K., \& Glow, H. (2012). "It"s not enough for the work of art to be great' : children and young people as museum visitors. Participations, 9(1), 26-42.

Koblin, A., \& Milk, C. (2013). This exquisite forest. In ACM SIGGRAPH 2013 Art Gallery (pp. 410-411). ACM. Retrieved from http://dl.acm.org/citation.cfm?id=2503669

Komiak, S., \& Benbasat, I. (2006). The Effects of Personalization and Familiarity on Trust and Adoption of Recommendation Agents. Retrieved from http://search.ebscohost.com/login.aspx?direct=true\&db=epref\&AN=BDAIEHFFI\&site=bsi-live

Liddle, D. E. (2006). The wider impact of Moore's law. Solid-State Circuits Society Newsletter, IEEE, 11(5), 28-30.

Linden, G., Smith, B., \& York, J. (2003). Amazon.com recommendations: Item-to-Item Collaborative Filtering. IEEE Internet Computing, $7(1), 76-80$.

Lindqvist, K. (2012). Museum finances: Challenges beyond economic crises. Museum Management and Curatorship, 27(1), 1-15.

Literat, I. (2012). The work of art in the age of mediated participation: Crowdsourced art and collective creativity. International Journal of Communication, 6, 23.

Lizardo, O. (2006). The puzzle of women's "highbrow" culture consumption: Integrating gender and work into Bourdieu's class theory of taste. Poetics, 34(1), 1-23.

McMahan, C., Hovland, R., \& McMillan, S. (2009). Online Marketing Communications. Journal of Interactive Advertising, 10(1), 61-76. http://doi.org/10.1080/15252019.2009.10722163

Proctor, N. (2011). The Google Art Project: A New Generation of Museums on the Web? Curator: The Museum Journal, 54(2), $215-221$.

Rayward, W. B., \& Twidale, M. B. (1999). From Docent to Cyberdocent: Education and Guidancein the Virtual Museum. Archives and Museum Informatics, 13(1), 23-53.

Rosenstein, C. (2010). When is a museum a public museum? Considerations from the point of view of public finance. International Journal of Cultural Policy, 16(4), 449-465. http://doi.org/10.1080/10286630902935178

Simon, N. (2010). The participatory museum. Museum 2.0. Retrieved from https://books.google.at/books?hl=ko\&lr=\&id=qun060 HU cOcC\&oi=fnd\&pg=PR1\&dq=TATE+museum+participatory+children\&ots=EdHbxaoWHi\&sig=Cse-M86_IWXXISIcionUf1gr31A 
Su, H., Comer, L., \& Lee, S. (2008). The effect of expertise on consumers' satisfaction with the use of interactive recommendation agents. Psychology and Marketing, 25(9), 859-880.

Tallon, L. (2008). Digital Technologies and the Museum Experience: Handheld Guides and Other Media. Rowman Altamira.

Wang, Y., Stash, N., Aroyo, L., Gorgels, P., Rutledge, L., \& Schreiber, G. (2008). Recommendations based on semantically enriched museum collections. Web Semantics: Science, Services and Agents on the World Wide Web, 6(4), 283-290.

Xiao, B., \& Benbasat, I. (2007). E-Commerce Product Recommendation Agents: Use, Characteristics, and Impact. MIS Quarterly, 31(1), 137-209. 